\title{
Hybrid Genetic Algorithm for Multi-Objective Transmission Expansion Planning
}

\author{
Phillipe Vilaça Gomes \\ INESC TEC and FEUP/DEEC
}

Dept. Eng. Eletrotécnica e de Computadores da Faculdade de Engenharia da Universidade do Porto

Porto, Portugal

phillipe.gomes@fe.up.pt

\begin{abstract}
This paper aims to describe a new tool to solve the Transmission Expansion Planning problem (TEP). The NonDominative CHA-Climbing Genetic Algorithm uses the standard blocks of Genetic Algorithms (GA) associated with an improvement of the population building block using Constructive Heuristic Algorithms (CHA) and Hill Climbing Method. TEP is a hard optimization problem because it has a non convex search space and integer and nonlinear nature, besides, the difficulty degree can be further increased if it includes more than one objective. In this work, a multi-objective TEP approach is detailed using an AC Optimal Power Flow to generate the set of Pareto solutions using the investment cost and the level of $\mathrm{CO}_{2}$ emissions, i.e. two conflicting objectives.
\end{abstract}

Index Terms - Transmission Expansion Planning, Multiobjective approach, Pareto solutions, AC Optimal Power Flow.

\section{INTRODUCTION}

The global warming in recent decades has been conducting several researchers from different areas to deepen their knowledge on mitigating the emission of greenhouse gases (GHG). According to the International Energy Agency (IEA), the electricity sector has a fundamental importance in this context [1], since it is responsible for about $40 \%$ of the $\mathrm{CO}_{2}$ emissions (in conjunction with heat). As shown in Fig. 1, it is therefore the sector responsible for the largest share of these emissions.

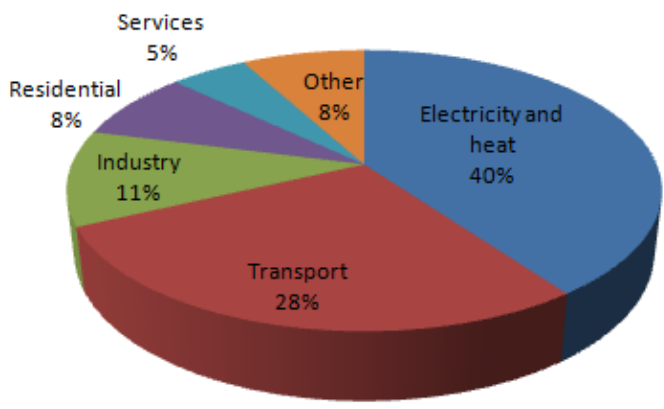

Figure 1: $\mathrm{CO}_{2}$ emissions by sector in 2013 (Source: IEA)

\author{
João Tomé Saraiva \\ INESC TEC and FEUP/DEEC \\ Dept. Eng. Eletrotécnica e de Computadores da \\ Faculdade de Engenharia da Universidade do Porto \\ Porto, Portugal \\ jsaraiva@fe.up.pt
}

The large amount of $\mathrm{CO}_{2}$ emissions associated with the electricity sector can be clearly understood analyzing the graph in Fig.2, in which the world power mix includes about $70 \%$ of power stations using fossil fuels [2].

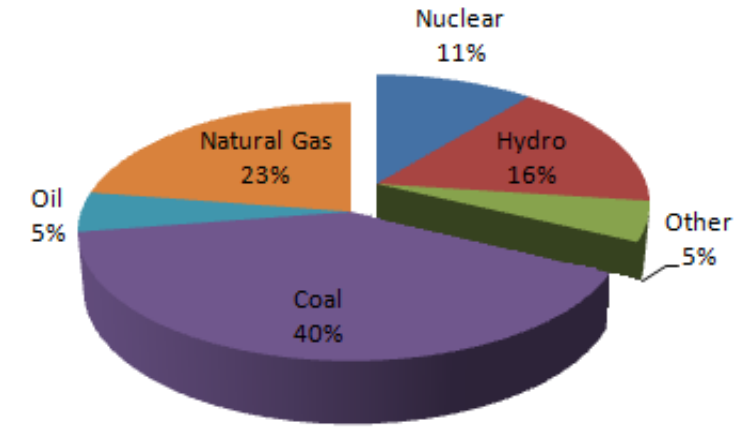

Figure 2: Fuel Share of Electricity Generation in 2012 (Source: IEA)

Therefore, it becomes trivial that the GHG mitigation challenge in the electricity sector is directed to the use of renewable energy sources rather than fossil fuels. Nevertheless in order to enable the increase of the penetration of renewable sources it becomes necessary, in many cases, the construction/ installation of new equipment (transmission lines, cables, transformers, etc) connecting the generating stations to the consumer centers, which means that a long-term transmission expansion planning exercise is in many cases required.

Transmission Expansion Planning (TEP) is one of the most challenging problems in power systems. The goal of this problem is the identification of the branches that should be reinforced or installed (or new paths to be built) and the most adequate schedule to expand them, to increase the power flow transmission capability and to alleviate network congestion. However, solving this problem is an extremely complex task since its search space has a discrete nature. Several solution approaches solve a relaxed continuous version of the original problem that is rounded at the end. This procedure does not ensure the identification of the global optimum. On the other hand, addressing the original discrete problem can lead to the explosion phenomenon in its search space. 
Generally, the TEP problem considers the expansion cost as its objective function to be minimized. However, it can also be using a multi-objective approach and that should attend, among others, to the following objectives:

- Alleviate transmission congestion;

- Minimize the risk associated to the investments;

- Minimize the investment and operation costs;

- Increase the reliability of the network;

- Increase the flexibility of system operation while reducing the network charges;

- Minimize the environmental impacts;

- Allow better voltage level regulation.

In this paper, the TEP problem was modelled with two conflicting objectives: the investment cost and the level of $\mathrm{CO}_{2}$ emissions. These objectives were considered in the scope of a new tool that is also detailed in this paper: The NonDominative CHA-Climbing Genetic Algorithm (NDCCGA). This tool includes the fundamental genetic algorithm blocks and an extra block to improve the population using Constructive Heuristic Algorithms (CHA) and Hill-Climbing (HC) techniques. Apart from that, it also includes a Tabu list to control the population diversity. This approach was developed using the $\mathrm{AC}$ power flow operation models, bearing in mind the gap between the AC and DC models [3]. Using this approach Transmission System Operators (TSO) will have more sounded information when analyzing possible solutions to the TEP problem, namely having different values of $\mathrm{CO}_{2}$ emissions and investments costs.

Regarding the structure of the paper, following this Introduction, Section II presents the AC model for the TEP problem and Section III provides a brief description of the multi-criteria approach. Section IV details the developed NDCCGA tool for this approach and Section V presents the results obtained in the simulations. Finally Section VI includes some comments and provides the conclusions about this work.

\section{MATHEMATICAL FoRMULATION OF THE TEP PROBLEM}

The AC model is the most adequate model to represent the operation conditions of the network in the scope of the TEP problem, because, it has the following main characteristics:

- It considers the reactive power;

- Losses are inherently included in the approach. If the DC model was used then an estimate of network losses had to be obtained for instance using the approach detailed in [4];

- It takes into account the voltage limits on the bars.

However, the use of the AC model leads to a complex nonlinear programming problem that requires an efficient optimization technique to be solved. The AC model was used in [5]-[7] to solve the TEP problem. In this paper the AC-OPF was conducted using the dispatch merit order related with the $\mathrm{CO}_{2}$ emissions of each power plant $\left(F_{1}\right)$, so that the planner is concerned with the minimization of these emissions thus leading to the AC-OPF given by (1) to (9).

$$
\operatorname{Min} F_{1}=\sum E_{i} \cdot P_{G i}
$$

subject to

$$
\begin{gathered}
P(V, \theta, n)-P_{G}+P_{D}=0 \\
Q(V, \theta, n)-Q_{G}+Q_{D}=0 \\
P_{G \text { min }} \leq P_{G} \leq P_{G \max } \\
Q_{G \text { min }} \leq Q_{G} \leq Q_{G \text { max }} \\
V_{\text {min }} \leq V \leq V_{\max } \\
(N+\stackrel{o}{N}) S^{\text {from }} \leq(N+\stackrel{o}{N}) S_{\text {max }} \\
(N+\stackrel{o}{N}) S^{t o} \leq(N+\stackrel{o}{N}) S_{\text {max }} \\
0 \leq n \leq n_{\text {max }}
\end{gathered}
$$

In this formulation $P(V, \theta, n)$ and $Q(V, \theta, n)$ are calculated by (10) and (11), and the bus conductance $G$ and susceptance B are given by (12) and (13).

$$
\begin{gathered}
V_{i} \sum V_{j}\left[G_{i j}(n) \cdot \cos \theta_{i j}+B_{i j}(n) \cdot \sin \theta_{i j}\right] \\
V_{i} \sum V_{j}\left[G_{i j}(n) \cdot \sin \theta_{i j}-B_{i j}(n) \cdot \cos \theta_{i j}\right] \\
G=\left\{\begin{array}{l}
G_{i j}(n)=-\left(n_{i j} \cdot g_{i j}+n_{i j} \cdot g_{i j}\right) \\
G_{i i}(n)=\sum_{j \in \Omega_{i}}\left(n_{i j} \cdot g_{i j}+n_{i j} \cdot g_{i j}\right.
\end{array}\right\} \\
B=\left\{\begin{array}{l}
B_{i j}(n)=-\left(n_{i j} \cdot b_{i j}+n_{i j} \cdot b_{i j}\right) \\
B_{i i}(n)=b_{i j}^{s h}+\sum_{j \in \Omega_{i}}\left[n_{i j}\left(b_{i j}+b_{i j}^{s h}\right)+n_{i j}\left(b_{i j}\right.\right. \\
\left.\left.b_{i j}+b_{i j}^{s h}\right)\right]
\end{array}\right\}
\end{gathered}
$$

The apparent flows $S^{\text {from }}$ and $S^{\text {to }}$ are calculated by (14) and (15) where $P_{i j}^{\text {from }}, Q_{i j}^{\text {from }}, P_{i j}^{t o}$ and $Q_{i j}^{t o}$ are given by (16) to (19).

$$
\begin{gathered}
S_{i j}^{\text {from }}=\sqrt{\left(P_{i j}^{\text {from }}\right)^{2}+\left(Q_{i j}^{\text {from }}\right)^{2}} \\
S_{i j}^{\text {to }}=\sqrt{\left(P_{i j}^{t o}\right)^{2}+\left(Q_{i j}^{t o}\right)^{2}} \\
P_{i j}^{\text {from }}=V_{i}^{2} \cdot g_{i j}-V_{i} \cdot V_{j}\left(g_{i j} \cdot \cos \theta_{i j}+b_{i j} \cdot \sin \theta_{i j}\right) \\
Q_{i j}^{\text {from }}=-V_{i}^{2} \cdot\left(b_{i j}^{\text {sh }}+b_{i j}\right)-V_{i} \cdot V_{j}\left(g_{i j} \cdot \sin \theta_{i j}-b_{i j} \cdot \cos \theta_{i j}\right) \\
P_{i j}^{t o}=V_{j}^{2} \cdot g_{i j}-V_{i} \cdot V_{j}\left(g_{i j} \cdot \cos \theta_{i j}-b_{i j} \cdot \sin \theta_{i j}\right) \\
Q_{i j}^{t o}=-V_{j}^{2} \cdot\left(b_{i j}^{\text {sh }}+b_{i j}\right)+V_{i} \cdot V_{j}\left(g_{i j} \cdot \sin \theta_{i j}+b_{i j} \cdot \cos \theta_{i j}\right)
\end{gathered}
$$

In the objective function (1) $E_{i}$ represents the $\mathrm{CO}_{2}$ emission rate for generator $\mathrm{i}, P_{G}$ is the real power generation, $Q_{G}$ is the reactive power generation, $P_{D}$ is the real power demand vector, $Q_{D}$ is the reactive power demand vector, $V$ is the voltage magnitude vector, $S^{t o, \text { from }}$ are the apparent power flow vectors in the branches in both terminals, $g_{i j}$ is the conductance in branch $\mathrm{i}-\mathrm{j}$ and $b_{i j}$ is the susceptance in 
branch i-j.

Apart from the level of $\mathrm{CO}_{2}$ emissions, each solution to the TEP problem is also characterized by the operation cost given by (20) and the investment cost calculated by (21). In these expressions $\alpha$ are coefficients of a generator cost function, $c_{i j}$ is the cost to install a network equipment in path $\mathrm{i}-\mathrm{j}$ and $\eta_{i j}$ is the number of equipments of that type to install in parallel in that path.

$$
\begin{gathered}
F_{2}=\sum \alpha_{i 1} \cdot P_{i}^{2}+\alpha_{i 2} \cdot P_{i}+\alpha_{i 3} \\
F_{3}=\sum c_{i j} \cdot n_{i j}
\end{gathered}
$$

As indicated before, the multi-objective problem is formulated using objectives F1 (level of emissions) and F3 (investment cost). In the current application, the operation cost is used to evaluate the feasibility of each solution because if a non-zero Power Not Supplied value occurs, then the F2 value is highly penalized and the corresponding solution is be most likely discarded in the scope of the Genetic Algorithm to be detailed in Section IV.

It is important to note that the DC model has been widely used because of the larger computational effort involved with the AC model. However, in recent years computer processors experienced an exponential technological advance, dramatically decreasing the processing time. In addition, some researchers reported a gap between DC based and AC models, even if heuristic approaches are used to cope with the increased complexity of AC models. According to [3] TEP solutions obtained using DC based models can significantly underestimate the expansion cost and are eventually unfeasible if they are analyzed using a complete $\mathrm{AC}$ power flow model.

\section{Multi-Criteria APPROACH}

As previously mentioned, TEP problem usually has more than one objective to be achieved. However the large majority of papers in the literature addresses the problem with only one goal. The multi-objective approach requires further analysis of the problem and the use of techniques that enable the approach since the problem presents a combinatorial nature that can easily lead to the well known the combinatorial explosion phenomenon of discrete problems.

In the approach described in this papers the objectives are the minimization of the investment $\operatorname{cost}\left(F_{3}\right)$ for a particular system expansion project and the minimization of the $\mathrm{CO}_{2}$ emissions $\left(F_{1}\right)$ during the operation horizon. So in addition to the transmission expansion planning, this approach allows to estimate the $\mathrm{CO}_{2}$ emissions level as well as the future operation costs.

The concept of dominance was used in the NDCCGA tool, for this particular case, with two objectives, the minimization of $F_{1}$ and the minimization of $F_{3}$. In this scope, a solution $x_{i}$ dominates a solution $x_{j}\left(x_{i} \triangleright,\right)$ when:

$$
x_{i} \triangleright \quad, \quad\left(x_{i}\right) \leq F_{1}\left(x_{j}\right) \wedge F_{3}\left(x_{i}\right) \leq F_{3}\left(x_{j}\right)
$$

As will be reported in the next section, the NDCCGA tool aims at building the set of non-dominated solutions, that is, the so called Pareto front.

\section{Non Dominative CHA-Climbing Genetic ALGORITHM TO BUILD THE PARETO Front}

The main blocks of the NDCCGA are similar to the ones of a genetic algorithm [8] applied to the solution of the TEP problem. Additionally, it includes an improvement population block and a Tabu list to control the diversity of the population. The population is composed of several individuals, each individual correspond to an expansion plan project in which each gene refers to the number of equipments to be constructed in a given path, and every individual is composed of a set of genes, as show in Fig. 3.

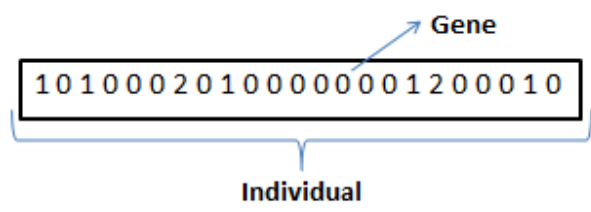

Figure 3: Representation in NDCCGA.

The initial population is randomly created and is ensured its diversity through a Tabu list. After this stage, the individuals will be evaluated according to the AC-OPF and each individual has its characteristics stored (expansion cost, operating cost, PNS and emission level).

Then, individuals will pass through the improvement block, in which individuals having PNS larger than a specified threshold will be improved by CHA and individuals having PNS within the allowed limits will be improved by the Hill Climbing method.

In the reproduction phase, different individuals are randomly grouped in pairs and their genes are exchanged in order to create two offspring. After that, these two offsprings are evaluated calculating the Operation Cost eventually penalized if PNS is not zero. The offspring having the lower Operation Cost passes to the next generation as a way to progressively discard solutions having non-zero PNS values.

The mutation occurs after the reproduction phase. An individual and then one of its genes are randomly chosen, and that gene is changed using the gene of another randomly selected individual. Then a new offspring population is created, in which, some individuals were mutated. Finally, the selection block classifies non-dominated individuals, i.e., the ones that are associated to non-dominated solutions using equation (22). If the number of the selected individuals, that is, the non-dominated solutions is smaller than the size of the population, then a tournament selection based on the operational costs is used to complete the population. This tournament selection also includes a Tabu list in order to ensure diversity. At the end of this step, the new population will be formed by the non-dominated individuals and the winners of the selection tournaments. This process is repeated until the convergence criterion is achieved or eventually the maximum number of iterations is run. 
The improvement block is based on the individual's characteristics, that is, if an individual has an unacceptable PNS, it is improved inserting new equipments selected using a CHA. On the other hand, if this individual displays an appropriate value to PNS, it is improved by eliminating equipments (change in the gene) using Hill Climbing Method. According to this method, an equipment is removed and the new individual is evaluated again. If the resulting PNS value continues appropriate this change is confirmed. If not, the mentioned equipment is included back in the individual.

CHAs are tools that have a low computational effort and that produce acceptable solutions [9], which makes their use interesting in hybridizing algorithms of the TEP problem. Basically, in each iteration these tools build a part of the solution, that is, new equipments are inserted in each iteration in order to progressively reduce or even eliminate PNS. The addition of new equipments occurs according to a preselected sensitivity criterion. In this case it was used the transited power flow, i.e, the addition is conducted in paths that display a larger congestion level.

The Hill Climbing method is a local search approach that starts with a solution and that tries to find better solutions in their neighborhood, through small changes in the current solution. In the present case, this tool checks if a particular equipment is crucial for the performance of that solution, i.e. if equipment is removed, the feasibility of the solution is checked in terms of having or not PNS. If the solution remains feasible, that is if PNS $=0$ the elimination of that equipment is confirmed. This technique requires the specification of the maximum number of individuals that will be analyzed (Max_Tries) as well as the maximum number of gene changings that are simulated (MaxFlips).

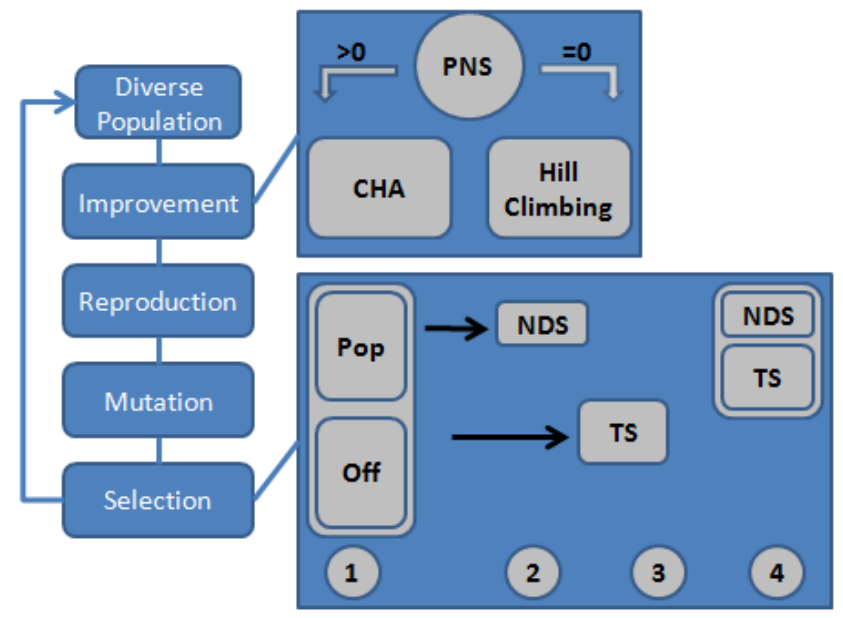

Figure 3: Overview of the application of the NDCCGA to the TEP problem.

Fig. 3 displays an overview of developed algorithm. In the selection block population (Pop) and offspring (Off) leads to the non-dominated solutions (NDS) and the tournament selection (TS) that incorporates the mentioned Tabu list to generate the new population.

It is worth emphasizing that the improvement block was introduced by the authors due to two factors: i. Genetic algorithms may have a high computational effort, i.e. require a large number of iterations to converge. On the other hand, when considering an improvement block to accelerate the convergence, the population diversity may be compromised because individuals may become local optima. The mentioned Tabu list was used to overcome these problems;

ii. When unfeasible solutions are modified introducing more equipments to regain feasibility, the generation dispatch becomes more flexible, that is, it is more likely to further reduce the level of emissions. On the other hand when removing some equipment the dispatch becomes less flexible and the level of emissions is likely to increase. Obviously, changing the number of equipments in the system also changes the investment cost. These changes are important in the scope of the iterative process detailed before because the investment cost and the level of emissions change eventually turning some solutions non-dominated. In particular, the extreme sides of the Pareto front may gain new solutions because including new equipments will increase the investment cost and conversely removing some equipments will reduce it.

\section{TESTS AND RESULTS}

The Non-Dominative CHA-Climbing Genetic Algorithm described in Section IV was applied on the modified IEEE 24 Bus Reliability Test System. The system used in the tests has some differences regarding the test system proposed in [10] as described below:

- Emergency Condition to the maximum allowed flow in a particular branch;

- To ensure the convergence of the AC-OPF, the loads were considered dispatchable as described in [11]. In this approach the loads are modeled as negative real power injection with associated negative costs. This modeling is performed using a negative output generator, ranging from a minimum injection equal to the negative total load to a maximum injection of zero. This means the problem has enough flexibility to reduce the demand if that is required to maintain feasibility. Additionally, if the entire load is not supplied the reactive demand is reduced in the same proportion as a way to keep the power factor of the original load.

- Reactive power sources are located in particular buses, as suggested in [12] and according to Table 1;

TABLE I. VOLTAGE CORRECTION DEVICES

\begin{tabular}{|c|c|c|c|}
\hline \multicolumn{4}{|c|}{ VOLTAGE CORRECTION DEVICES } \\
\hline DEVICE & BUS & MVAR & CAPABILITY \\
\hline $\begin{array}{c}\text { SYNCH. } \\
\text { CONDENSER }\end{array}$ & 3 & 350 & CAPACITIVE \\
\hline $\begin{array}{c}\text { SYNCH. } \\
\text { CONDENSER }\end{array}$ & 9 & 510 & CAPACITIVE \\
\hline
\end{tabular}

- The values of all loads and of the installed capacity of all generators were tripled (real and reactive) in order to turn the network more stressed. 
According to Section IV, NDCCGA was used to build the Pareto front integrating solutions that reflect a trade-off between the minimization of Emissions (F1) and Investment Costs (F3). This new tool was used with a population of 1000 individuals, penalty factor for PNS equals to $10^{7} € / \mathrm{MW}$ and the cost of dispatchable loads was set at $10^{6} € / \mathrm{MW}, 1 \mathrm{MW}$ for allowed PNS, the MaxTries equals to 30 and 50 for MaxFlips. The voltage limit on the bars is $5 \%$ (0.95 and 1.05 p.u). The iterative process finishes when the solutions in the Pareto Front obtained in two consecutive iterations do not change more than a specified threshold. In the simulations to be detailed below convergence was obtained after running 300 iterations because from that point onwards the changes in the Pareto Front were neglectable.

The developed software converged in about 34 hours, solving 969.148 AC-OPFs, the NDCCGA was implemented in MATLAB, running on an Intel i7, 3.4GHz, 8 GB RAM, hardware platform and the AC-OPFs was solved with interior point solver using MATPOWER tool described in [11].

Figure 3 presents the Pareto front of the TEP problem. Solution 8 has the largest investment cost and the lowest CO2

TABLE II. emissions. On the other hand, solution 1 presents the lowest investment cost and the largest $\mathrm{CO} 2$ emissions. The other solutions indicated in Fig. 3 are detailed in the Table 2.

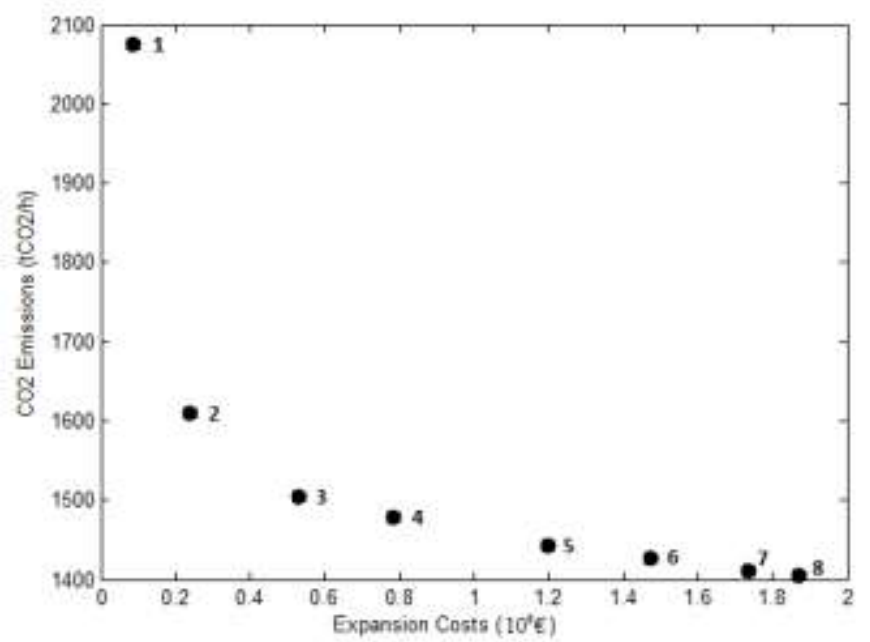

Figure 3: Pareto Front for minimizing Investment costs and $\mathrm{CO}_{2}$ emissions.

\begin{tabular}{|c|c|c|c|c|}
\hline Solution & Expansion Planning (path) & $\begin{array}{c}\text { Operational } \\
\text { costs }(€ / \mathbf{h})\end{array}$ & $\begin{array}{l}\text { Emissions } \\
(\mathrm{tCO} 2 / \mathrm{h})\end{array}$ & $\begin{array}{l}\text { Expansion } \\
\text { Costs } \\
\left(10^{9} €\right)\end{array}$ \\
\hline 1 & $138 \mathrm{kV}$ cable (06-10), $138 \mathrm{kV}$ line (07-08) and $230 \mathrm{kV}$ line (14-16) & $1,7494.10^{6}$ & 2075,17 & 0,086 \\
\hline 2 & $\begin{array}{l}138 \mathrm{kV} \text { cable }(06-10), 138 \mathrm{kV} \text { line }(07-08), 230 \mathrm{kV} \text { line }(11-13), 230 \mathrm{kV} \text { line }(15-21) \\
\text { and } 230 \mathrm{kV} \text { line }(15-24) .\end{array}$ & $1,7690.10^{6}$ & 1610,27 & 0,2380 \\
\hline 3 & $\begin{array}{l}138 \mathrm{kV} \text { cable }(06-10), 138 \mathrm{kV} \text { line }(07-08) \text {, Transformer }(10-12), 230 \mathrm{kV} \text { line }(11- \\
13), 230 \mathrm{kV} \text { line }(12-23), 230 \mathrm{kV} \text { line }(15-21), 230 \mathrm{kV} \text { line }(16-17) \text { and } 230 \mathrm{kV} \text { line } \\
(17-22) .\end{array}$ & $1,7521.10^{6}$ & 1505,36 & 0,532 \\
\hline 4 & $\begin{array}{l}138 \mathrm{kV} \text { cable }(06-10), 138 \mathrm{kV} \text { line }(07-08) \text {, Transformer }(10-11),(2) 230 \mathrm{kV} \text { line } \\
(11-13),(2) 230 \mathrm{kV} \text { line }(12-23),(2) 230 \mathrm{kV} \text { line }(15-21), 230 \mathrm{kV} \text { line }(15-24) \text { and } \\
230 \mathrm{kV} \text { line }(21-22) .\end{array}$ & $1,7473.10^{6}$ & 1479,08 & 0,784 \\
\hline 5 & $\begin{array}{l}\text { (2) } 138 \mathrm{kV} \text { cable }(01-02) \text {, Transformer }(03-24),(2) 138 \mathrm{kV} \text { line }(04-09),(2) 138 \mathrm{kV} \\
\text { line }(05-10),(2) 138 \mathrm{kV} \text { line }(07-08),(2) 138 \mathrm{kV} \text { line }(08-10),(2) \text { Transformer }(10- \\
\text { 11), Transformer }(10-12), 230 \mathrm{kV} \text { line }(11-14), 230 \mathrm{kV} \text { line }(13-23), 230 \mathrm{kV} \text { line }(15- \\
\text { 16), } 230 \mathrm{kV} \text { line }(16-19), 230 \mathrm{kV} \text { line }(17-22),(2) 230 \mathrm{kV} \text { line }(18-21),(2) 230 \mathrm{kV} \\
\text { line }(19-20),(4) 230 \mathrm{kV} \text { line }(20-23) \text { and } 230 \mathrm{kV} \text { line }(21-22) .\end{array}$ & $1,7406.10^{6}$ & 1443,09 & 1,200 \\
\hline 6 & $\begin{array}{l}138 \mathrm{kV} \text { line }(04-09), 138 \mathrm{kV} \text { line }(05-10), 138 \mathrm{kV} \text { cable }(06-10),(2) 138 \mathrm{kV} \text { line }(07- \\
08) \text {, Transformer }(10-12), 230 \mathrm{kV} \text { line }(11-13),(5) 230 \mathrm{kV} \text { line }(12-23), 230 \mathrm{kV} \text { line } \\
(14-16), 230 \mathrm{kV} \text { line }(11-13),(2) \quad 230 \mathrm{kV} \text { line }(15-21), 230 \mathrm{kV} \text { line }(15-24), \\
230 \mathrm{kV} \text { line }(16-17) \text { and (2) } 230 \mathrm{kV} \text { line } \quad(17-22) .\end{array}$ & $1,7375.10^{6}$ & 1426,45 & 1,474 \\
\hline 7 & $\begin{array}{l}\text { Transformer }(03-24), 138 \mathrm{kV} \text { line }(04-09), 138 \mathrm{kV} \text { line }(05-10), 138 \mathrm{kV} \text { cable }(06- \\
10),(2) 138 \mathrm{kV} \text { line }(07-08) \text {, Transformer }(10-12), 230 \mathrm{kV} \text { line }(11-13),(5) 230 \mathrm{kV} \\
\text { line }(12-23), 230 \mathrm{kV} \text { line }(14-16), 230 \mathrm{kV} \text { line }(11-13),(2) 230 \mathrm{kV} \text { line }(15-21), 230 \\
\mathrm{kV} \text { line }(15-24),(2) 230 \mathrm{kV} \text { line }(16-17),(3) 230 \mathrm{kV} \text { line }(17-22) \text { and } 230 \mathrm{kV} \text { line } \\
(20-23) .\end{array}$ & $1,7348.10^{6}$ & 1411,81 & 1,736 \\
\hline 8 & $\begin{array}{l}\text { Transformer }(03-24), 138 \mathrm{kV} \text { line }(04-09), 138 \mathrm{kV} \text { line }(05-10), 138 \mathrm{kV} \text { cable }(06- \\
10),(2) 138 \mathrm{kV} \text { line }(07-08) \text {, Transformer }(10-12), 230 \mathrm{kV} \text { line }(11-13),(5) 230 \mathrm{kV} \\
\text { line }(12-23), 230 \mathrm{kV} \text { line }(14-16), 230 \mathrm{kV} \text { line }(11-13),(3) 230 \mathrm{kV} \text { line }(15-21) \text {, } \\
230 \mathrm{kV} \text { line }(15-24),(2) 230 \mathrm{kV} \text { line }(16-17),(3) 230 \mathrm{kV} \text { line }(17-22),(2) \\
230 \mathrm{kV} \text { line }(20-23) \text { and (2) } 230 \mathrm{kV} \text { line }(21-22) .\end{array}$ & $1,7336.10^{6}$ & 1405,39 & 1,870 \\
\hline
\end{tabular}


Therefore, this approach provides the set of solutions among which the TSO will select the one to expand the network according to values obtained for Functions F1, F2 and F3 and also having in mind limits for instance related with the maximum investment cost, GHG emission reduction agreements, etc.

\section{CONCLUSIONS}

This paper presents a new tool able to deal with multiobjective Transmission Expansion Planning problems. The multi-objective formulation includes the reduction of the $\mathrm{CO}_{2}$ emissions (given that there is a growing concern because of its impact on global warming) and the minimizing of the investment costs in network equipments.

The Non-Dominative CHA-Climbing Genetic Algorithm has the general blocks of a regular genetic algorithm with the addition of an improvement block and a Tabu list. The improvement is made using Constructive Heuristic Algorithms and Hill Climbing method. Each candidate solution is analyzed using an $\mathrm{AC}-\mathrm{OPF}$ model that was preferred to $\mathrm{DC}$ based versions in view of the existing gap between these two models.

The NDCCGA was applied to the IEEE 24-Bus Reliability Test System modified with the inclusion of two voltage control devices and the network operating conditions were stressed tripling the generation capacities and the loads, which in turn were considered as dispatchable. The tool showed excellent performance providing a number of different expansion plans with different values of $\mathrm{CO}_{2}$ emissions and investment costs. This enables the decision maker to select the final one in a more informed way.

The main contribution of this paper is the development of NDCCGA tool that provides to the end of its iterative process a tradeoff between investment cost and emissions without the individual and separate minimization, as in other approaches. This process proved to be beneficial since it saves computational effort, which in this case enabled using a more complete AC-OPF model.

\section{ACKNOWLEDGEMENTS}

The first author thanks CAPES Foundation, Ministry of Education of Brazil, for financing this research and Dr. Suzana Menezes de Souza for the several teachings provided in the INESC TEC premises.

\section{REFERENCES}

[1] International Energy Agency, "Recent trends in OECD CO2 emissions from fuel combustion," 2015.

[2] I. E. Agency, "Key World Energy Statistics," 2014.

[3] R. Bent, C. Coffrin, R. R. E. Gumucio, and P. Van Hentenryck, "Transmission Network Expansion Planning: Bridging the gap between AC heuristics and DC approximations," in 2014 Power Systems Computation Conference, 2014, pp. 1-8.

[4] M. C. da Rocha and J. T. Saraiva, "A discrete evolutionary PSO based approach to the multiyear transmission expansion planning problem considering demand uncertainties," Int. J. Electr. Power Energy Syst., vol. 45, no. 1, pp. 427-442, Feb. 2013.

[5] T. Akbari, A. Rahimi-Kian, and M. Tavakoli Bina, "Security-constrained transmission expansion planning: A stochastic multi-objective approach," Int. J. Electr. Power Energy Syst., vol. 43, no. 1, pp. 444453, Dec. 2012.

[6] T. Akbari, M. Tavakoli Bina, and A. Abedini, "ACOPF based static transmission expansion planning: A multi-objective approach," in 20th Iranian Conference on Electrical Engineering (ICEE2012), 2012, pp. 364-369.

[7] M. Moghaddam, H. Abdi, and M. Javidi, "Transmission Expansion Planning in Competitive Electricity Markets Using AC OPF," in 2006 IEEE PES Power Systems Conference and Exposition, 2006, pp. 1507-1512.

[8] A. Sadegheih and P. R. Drake, "System network planning expansion using mathematical programming, genetic algorithms and tabu search," Energy Convers. Manag., vol. 49, no. 6, pp. 15571566, Jun. 2008.

[9] P. V. Gomes and J. T. Saraiva, "Static transmission expansion planning using Heuristic and metaheuristic techniques," in 2015 IEEE Eindhoven PowerTech, 2015, pp. 1-6.

[10] P. Subcommittee, "IEEE Reliability Test System," IEEE Trans. Power Appar. Syst., vol. PAS-98, no. 6, pp. 2047-2054, Nov. 1979.

[11] R. D. Zimmerman, C. E. Murillo-Sanchez, and R. J. Thomas, "MATPOWER: Steady-State Operations, Planning, and Analysis Tools for Power Systems Research and Education," IEEE Trans. Power Syst., vol. 26, no. 1, pp. 12-19, Feb. 2011.

[12] M. J. Rider, A. V. Garcia, and R. Romero, "Power system transmission network expansion planning using AC model," IET Gener. Transm. Distrib., vol. 1, no. 5, p. 731, Sep. 2007. 